\title{
Una nueva mirada a los determinantes del peso infantil en la primera infancia
}

\section{A new examination of the determinants of weight in early childhood}

\author{
Viviana Salinas ${ }^{a}$, Jael Goldsmith Weil ${ }^{\mathrm{b}}$
}

alnstituto de Sociología, Pontifica Universidad Católica de Chile y Núcleo Milenio para el Estudio del Curso de Vida y la Vulnerabilidad. Santiago, Chile

${ }^{b}$ Centro de Estudios del Desarrollo Regional y Políticas Públicas Ceder, Universidad de los Lagos. Osorno, Chile

Recibido: 28 de junio de 2019; Aceptado: 25 de agosto de 2020

\section{¿Qué se sabe del tema que trata este estudio?}

La obesidad infantil en Chile no se distribuye de forma uniforme, sino que hay una mayor concentración de sobrepeso en sectores con menor nivel socioeconómico.

\section{¿Qué aporta este estudio a lo ya conocido?}

Ingreso y escolaridad materna tienen efectos heterogéneos sobre peso infantil según edad y estatus nutricional. En niños de 5-6 años, mayor ingreso se asocia a mayor obesidad si las madres tienen baja escolaridad. Lo contrario ocurre si tienen alta escolaridad.

\section{Resumen}

En las últimas décadas, Chile ha experimentado una triple transición demográfica, nutricional y económica. Objetivo: Explorar la relación entre peso infantil y dos aspectos del nivel socioeconómico, ingreso familiar y educación materna, estudiando el efecto de cada uno por si solo y la manera en que operan juntos para determinar el peso infantil y comparar su efecto en menores obesos y en otro estatus de peso. Sujetos y Método: Estudio de fuentes secundarias de datos obtenidos de la Encuesta Longitudinal de Primera Infancia de 2012, analizando niños y niñas entre 2 y 6 años de edad $(\mathrm{n}=11.339)$. Se estimaron modelos multivariados de regresión cuantílica para el puntaje $\mathrm{z}$ del índice de masa corporal (zIMC). Resultados: En niños, entre 2 y 3 años, el ingreso y peso infantil tuvieron una asociación positiva, mientras que educación materna y peso infantil una negativa. Entre los 4 y 6 años, ingreso y peso infantil tuvieron una asociación negativa entre hijos de madres con estudios superiores, pero positiva entre hijos de madres con más baja educación. Conclusión: Ingreso familiar y educación materna tienen efectos opuestos en el peso infantil. El efecto positivo de ingreso sobre zIMC es mitigado en casos de madres con altos logros educativos. Recomendamos estudiar el efecto de ingreso y educación en el peso infantil por separado e investigar los mecanismos causales que explican la relación entre determinantes socioeconómicos y peso infantil.

\section{Palabras clave:}

Peso;

Nivel Socioeconómico;

Obesidad Infantil;

Chile;

Índice de Masa

Corporal

Correspondencia:

Viviana Salinas

vmsalina@uc.cl 


\begin{abstract}
In the last decades, Chile has experienced a triple transition regarding demographic, nutritional, and economic issues. Objective: To explore the relationship between childhood weight and two dimensions of socioeconomic status, family income, and maternal educational level, analyzing the effect of each one by itself and how they operate together to determine childhood weight and comparing their effect on obese and non-obese children. Subjects and Method: Study based on data from the 2012 Encuesta Longitudinal de Primera Infancia (Early Childhood Longitudinal Survey), evaluating children between 2 and 6 years old $(n=11,399)$. We estimated multivariate quantile regression models for the z-score of the body mass index (BMI-z). Results: We found that in children aged 2-3 years, income and weight had a positive association, while maternal educational level and weight had a negative one. In children aged 4-6 years, income and weight were negatively associated among children whose mothers have a higher educational level but positive among those with lower educational levels. Conclusion: Family income and maternal educational level have opposite effects on childhood weight. The positive effect of income on BMI-z is diminished when mothers have high educational levels. We recommend studying the effects of income and education on child weight separately and exploring the causal mechanisms that explain the relations between socioeconomic determinants and childhood weight.
\end{abstract}

Keywords:

Body Weight;

Socioeconomic Status;

Child Obesity;

Chile;

Body Mass Index

\section{Introducción}

Desde mediados del siglo XX al presente, Chile ha experimentado una triple transición demográfica, nutricional y económica. La desnutrición infantil pasó de $37 \%$ en 1960 a estar virtualmente erradicada ${ }^{1}$. En 2018, 50,9\% del total de niños y niñas medidos por el mapa nutricional JUNAEB estaban en un estado de malnutrición por exceso (sobrepeso y obesidad) ${ }^{2}$. La mortalidad infantil pasó de 241 por mil nacidos vivos en 1939 a 6,6 en $2017^{3}$. El país experimentó un rápido crecimiento económico, desde el subdesarrollo a incorporarse a la Organización para la Cooperación y Desarrollo Económico en 2010. También se transformaron los ambientes alimentarios, con mejoras en inocuidad alimentaria y aumento en disponibilidad y consumo de alimentos procesados (altos en calorías y deficientes en nutrientes) ${ }^{4,5}$. En conjunto, estos cambios modernizantes nos ubican en un momento de la transición nutricional donde se supera la desnutrición por escasez y se generan enfermedades degenerativas relacionadas a la obesidad ${ }^{3,6-12}$.

La obesidad infantil en Chile no se distribuye de forma uniforme, sino que hay una mayor concentración de sobrepeso en sectores con menor nivel socioeconómico (NSE) ${ }^{13,14}$. Aunque se puede constatar esta correlación, no existen certezas sobre los mecanismos por los cuales el contexto socioeconómico influye en obesidad infantil. Tampoco hay evidencia acerca de si los determinantes de obesidad infantil actúan de igual manera en niños y niñas con y sin sobrepeso en Chile, ni antecedentes sobre estudios que apliquen un análisis desagregado en todo el espectro de IMC durante la primera infancia. En este artículo, se exploran los determinantes del peso en primera infancia en Chile, analizando dos aspectos del NSE, el ingreso familiar y educación de la madre, tanto de forma desagregada como conjunta, y se compara su efecto en menores obesos y en otro estatus de peso.

\section{Sujetos y Método}

Estudio de fuentes secundarias. Los datos provienen de la Encuesta Longitudinal de Primera Infancia (ELPI). Esta encuesta se inició en 2010, teniendo como marco muestral a los niños nacidos entre el $1^{\circ}$ de enero de 2006 y el $31^{\circ}$ de agosto de 2009. En la segunda ola, de 2012, se agregó una muestra complementaria de niños nacidos entre el $1^{\circ}$ de septiembre de 2009 y el $31^{\circ}$ de diciembre de 2011. El marco muestral para la selección de los sujetos provino de datos del Servicio de Registro Civil e Identificación. El diseño muestral, en ambas olas fue estratificado, bietápico. Los estratos fueron construidos conglomerando comunas de similar nivel socioeconómico, para lo que se utilizó información Censo de Población de 2002 y de la Encuesta Casen de 2006. En la primera etapa se seleccionaron comunas (las comunas con más de 60.000 habitantes y todas las de del Gran Santiago fueron seleccionadas con probabilidad 1, por el tamaño de su población). En la etapa dos se seleccionaron casos a investigar a través de muestreo aleatorio sistemático. Este diseño muestral, en dos etapas, suele ser elegido porque permite obtener una muestra representativa de la población en un tiempo corto y a menor costo. La distribución de la muestra se realizó de forma proporcional a la población de cada estrato considerando las 15 regiones del 
país ${ }^{15}$. La Encuesta cuenta con factores de expansión transversal, que combinan la probabilidad total de selección del individuo definida por el diseño muestral (considerando que el tipo de muestreo utilizado y la forma de selección de las unidades de primera y segunda etapa) con el factor de expansión de cada individuo seleccionado en cada comuna seleccionada (que es el recíproco de probabilidad total de selección del individuo) y un componente de ajuste (definido como el cuociente entre la muestra objetivo y la muestra lograda en cada estrato, considerando que, como ocurre típicamente en encuestas de hogares, en algunos estratos se obtuvo una cantidad de encuestas menor a las determinadas en el diseño original $)^{15}$. Sin embargo, esta investigación no utilizó dichos factores de expansión. Dicho de otra manera, todos los análisis en este estudio son no-ponderados. Aunque usar los factores de expansión habría proporcionado guarismos de tamaño similar al tamaño de la población (que es el objetivo de usar factores de expansión), los estimadores de parámetros poblacionales que se obtienen sin ponderar no solo son insesgados y consistentes, sino que generalmente son más eficientes que sus contrapartes ponderadas $^{16,17}$.

En esta investigación, se utilizaron solamente los datos recogidos en 2012 de manera transversal. Se aplicó un cuestionario al cuidador principal y pruebas psicológicas y mediciones antropométricas a los niños seleccionados y a su cuidador principal. La institución que recogió los datos obtuvo consentimiento informado firmado del cuidador principal. Siguiendo las recomendaciones de la Organización Mundial de la Salud (OMS) que advierten que el índice de masa corporal (IMC) no es una medición adecuada para determinar el estado nutricional en lactantes ${ }^{18}$, se excluyeron los menores de dos años de edad de este análisis.

Con los datos antropométricos de los niños se calculó el puntaje z del IMC (ZIMC), usando el software WHO Anthro para menores de hasta 60 meses de edad y WHO Anthro Plus, para mayores de 60 meses $^{19}$. Se clasificó a sujetos según su ZIMC siguiendo criterios de la $\mathrm{OMS}^{18}$ : desnutrición, $<-2$; peso normal, $=-2$ y $<1$; sobrepeso, $=1 \mathrm{y}<2$; y obesidad, $>=2$. Se calculó el IMC materno, distinguiendo madres obesas (IMC $>=30$ ) de no obesas.

Del cuestionario al cuidador principal se obtuvieron dos indicadores de NSE: ingreso per cápita del hogar y nivel educacional de la madre. Este último se utilizó como variable dicotómica, distinguiendo a madres con estudios superiores (técnicos o universitarios, completos e incompletos), de madres con menos educación. El ingreso se incorporó como logaritmo del ingreso, considerando su distribución no normal, debido a que la mayor parte de la muestra tiene ingresos más bien bajos, concretándose en la parte izquierda de la distribución, y solamente un porcentaje pequeño tiene ingresos altos, dando origen a una cola larga hacia la derecha. Además, se incluyó en el análisis multivariado: sexo del menor, edad en meses, residencia rural o urbana y pertenencia a etnia indígena (reportado por la madre). También se controló por obesidad materna (calculada en base al autoreporte de peso y estatura de las madres y su correspondiente IMC), prácticas de lactancia (amamantados por más de seis meses) y consumo de alimentos saludables. Para este último, se siguió el ejemplo de Marteleto y cols. ${ }^{20}$ construyendo un índice que sirve de proxy de consumo a partir de preguntas sobre la frecuencia de consumo de diversos alimentos del niño. El índice se construyó a partir de un análisis factorial (policórico, por la naturaleza no continua de las categorías de respuesta de los ítems). Los ítems que entraron al análisis factorial fueron: completos, hamburguesas, pizzas, papas fritas, etc.; carnes o pescados; legumbres; pan, tallarines, arroz; chocolates o dulces jugos o bebidas envasadas; papas fritas, suflitos u otros snacks en bolsa; leche; agua; galletas; frutas y verduras. Las variables con más peso en el análisis fueron leche, agua y frutas y verduras, por lo que se considera al factor emergente como indicador de comida saludable. Luego de construido el índice, se lo dicotomizó, distinguiendo a menores con un alto consumo de alimentos saludables (25\% superior del índice).

Los valores perdidos (es decir, los casos en que los encuestados no respondieron) fueron: para zIMC, $8,4 \%$ de los casos; obesidad materna, $1 \%$; educación materna, 2,3\%; consumo de alimentos saludables, 0,4\%; e ingreso per cápita del hogar, 2,1\%. Se realizó un análisis de casos completos y se excluyeron los casos en que el cuidador principal no era la madre $(1,7 \%)$. El tamaño final de la muestra fue 11.339. Se analizó por separado a niños entre 2 y 3 años (24 a 47 meses) y de 4 a 6 años (48 a 83 meses). Esta estratificación se hizo teniendo en cuenta que un porcentaje importante de niños ingresan al sistema preescolar a los cuatro años, lo que puede significar un cambio en actividad física y hábitos alimentarios, por tanto, se consideró un adecuado punto de corte.

Se realizó un análisis multivariado con modelos de regresión cuantílica. A diferencia de la regresión lineal, que estima el efecto de una serie de predictores sobre el promedio de una variable dependiente, la regresión cuantílica estima el efecto de los predictores en diferentes puntos de la distribución de la variable dependien$\mathrm{te}^{21}$. Esto es pertinente no solo porque el IMC tiene diferentes significados en diferentes puntos de su distribución, sino también porque enfocarse en el promedio puede llevar a errores en sociedades que atraviesan una transición nutricional, donde los valores del IMC pueden cambiar rápidamente y la asociación entre IMC y sus predictores puede ser diferente a lo largo del es- 
pectro del $\mathrm{IMC}^{11}$. Se usaron cuatro percentiles (p): p5, p50, p85 y p95, que son los criterios con que el Centro de Control y Prevención de Enfermedades de los Estados Unidos (CDC) define bajo peso, normo-peso, sobrepeso y obesidad infantil en niños(as) mayores a 2 $\operatorname{años}^{22,23}$ y que han sido utilizados en investigación chilena previamente ${ }^{13,14}$. Los modelos se estimaron también usando los criterios p3 y p97. Los resultados son en general consistentes y están disponibles a solicitud.

El primer modelo incluyó las dos medidas de NSE, obesidad materna, consumo de alimentos saludables, prácticas de lactancia, sexo, edad, identificación étnica y residencia rural. El segundo modelo consideró las mismas variables, pero incluyo además la interacción entre ingreso familiar y educación de la madre, para evaluar diferencias en el efecto del ingreso entre madres con y sin estudios superiores y entender mejor el efecto del NSE en peso infantil. Se calcularon los efectos marginales de este modelo de modo de hacer más comunicable la acción combinada de educación materna e ingreso familiar. Los efectos marginales son una medida del valor predicho en el modelo para individuos que tienen determinadas características. En este caso, se obtuvo el valor predicho o esperado del ZIMC para hijos(as) de madres con y sin educación superior y en los diferentes niveles de ingreso familiar. Esos valores esperados con los que se grafican, de manera que pueda verse como cambia el zIMC antes diferentes niveles de ingreso familiar en el caso de los hijos(as) de madres con educación superior, por una parte, y sin educación superior, por otra. Las demás variables del modelo quedaron en su valor promedio, es decir, los valores graficados describen a niños(as) promedio.

\section{Resultados}

La tabla 1 resume las características de la muestra. La mitad de los niños tuvieron exceso de peso y solo el $1 \%$ presentó desnutrición. La muestra estuvo balanceada en términos de sexo y un $10 \%$ de las madres clasificaron a su hijo como perteneciente a un pueblo originario. La mayor parte de los niños vivía en zonas urbanas. Un 18\% de las madres tenía estudios postsecundarios y un $30 \%$ de ellas tuvo algún grado de obesidad. La distribución de todas las variables fue similar en ambos tramos etarios.

El análisis multivariado para todos los sujetos entre 2 y 3 años se resumió en la tabla 2 . En este grupo etario, la interacción entre educación materna y logaritmo del ingreso (Modelo 2) no fue significativa, por lo que se presenta solo el Modelo 1. Las dos variables que medían NSE fueron estadísticamente significativas, pero el efecto de educación materna fue negativo, mientras que el de logaritmo del ingreso fue positivo. En cuanto a educación materna, el ZIMC fue más bajo en hijos de madres con educación superior en comparación a madres con menor educación. Este efecto fue significativo entre menores con sobrepeso y obesidad (p85 y p95), pero no entre los que tenían normo o bajo peso. El logaritmo del ingreso tuvo un efecto positivo sobre el ZIMC (mayor ingreso familiar se asocia a mayor ZIMC) entre niños del p50 y p85 (peso normal y sobrepeso). El efecto no fue significativo entre menores obesos ni de bajo peso.

Respecto de los demás predictores, la obesidad materna tuvo un efecto positivo y grande (el de mayor magnitud de todas los variables incorporadas en

Tabla 1. Descripción de la muestra, niños chilenos de 2 a 6 años, 2012

\begin{tabular}{|c|c|c|c|}
\hline Variables & 2 a 3 años & 4 a 6 años & Total \\
\hline $\mathrm{N}$ & 4.492 & 6.847 & 11.339 \\
\hline Edad (meses) & $38,7 \pm 6,4$ & $63,4 \pm 8,8$ & $53,6 \pm 14,5$ \\
\hline \multicolumn{4}{|l|}{ Estado nutricional (\%) } \\
\hline Desnutrición & 1,3 & 0,8 & 1,0 \\
\hline Peso normal & 44,8 & 47,4 & 46,3 \\
\hline Sobrepeso & 32,4 & 31,8 & 32,0 \\
\hline Obesidad & 21,7 & 20,1 & 20,7 \\
\hline Mujeres (\%) & 48,8 & 51,2 & 50,3 \\
\hline Afiliación indígena (\%) & 9,6 & 9,9 & 9,8 \\
\hline Residencia rural (\%) & 10,6 & 11,3 & 11,0 \\
\hline Madres estudios superiores (\%) & 18,2 & 18,5 & 18,4 \\
\hline Ingreso del hogar promedio, per cápita (desviacion estandar) & $118,4(119,5)$ & $115,8(113,6)$ & $116,8(116,0)$ \\
\hline Madre obesa (\%) & 28,7 & 30,0 & 29,5 \\
\hline Lactancia de más de seis meses (\%) & 66,8 & 68,0 & 67,5 \\
\hline
\end{tabular}


Tabla 2. Modelos de Regresión Cuantílica del Puntaje Z del Índice de Masa Corporal, Niños Chilenos entre 24 y 48 meses ( 2 y 4 años)

\begin{tabular}{|c|c|c|c|c|}
\hline & $\begin{array}{c}\text { p5 } \\
\text { (bajo peso) }\end{array}$ & $\begin{array}{c}\text { p50 } \\
\text { (normopeso) }\end{array}$ & $\begin{array}{c}\text { p85 } \\
\text { (sobrepeso) }\end{array}$ & $\begin{array}{c}\mathrm{p95} \\
\text { (obesidad) }\end{array}$ \\
\hline N & 4,492 & 4,492 & 4,492 & 4,492 \\
\hline \multirow[t]{2}{*}{ Madre con educación superior } & $-0,06$ & $-0,08$ & $-0,19 * * *$ & $-0,20 * *$ \\
\hline & $(0,15)$ & $(0,06)$ & $-0,07$ & $(0,10)$ \\
\hline \multirow[t]{2}{*}{ Logaritmo del ingreso familiar per cápita } & 0,10 & $0,05^{*}$ & $0,10 * *$ & 0,05 \\
\hline & $(0,07)$ & $(0,02)$ & $(0,04)$ & $(0,05)$ \\
\hline \multirow[t]{2}{*}{ Madre obesa } & 0,23 & $0,29 * * *$ & $0,37 * * *$ & $0,24 * *$ \\
\hline & $(0,15)$ & $(0,05)$ & $(0,07)$ & $(0,10)$ \\
\hline \multirow[t]{2}{*}{ Alto consumo alimentos saludables } & 0,01 & $-0,06$ & $-0,08$ & $-0,03$ \\
\hline & $(0,07)$ & $(0,04)$ & $(0,06)$ & $(0,07)$ \\
\hline \multicolumn{5}{|l|}{ Lactancia mayor a 6 meses } \\
\hline \multirow[t]{2}{*}{ Edad en meses } & 0,00 & $-0,02$ & $-0,00$ & 0,00 \\
\hline & $(0,00)$ & $(0,01)$ & $(0,00)$ & $(0,01)$ \\
\hline \multirow[t]{2}{*}{ Sexo femenino } & $-0,01$ & $-0,04$ & $-0,05$ & $-0,14 *$ \\
\hline & $(0,10)$ & $(0,04)$ & $(0,06)$ & $(0,09)$ \\
\hline \multirow[t]{2}{*}{ Indígena } & $-0,13$ & 0,01 & 0,08 & 0,11 \\
\hline & $(0,19)$ & $(0,08)$ & $(0,10)$ & $(0,15)$ \\
\hline \multirow[t]{2}{*}{ Rural } & 0,13 & $-0,03$ & $-0,05$ & $-0,24^{*}$ \\
\hline & $(0,17)$ & $(0,07)$ & $(0,07)$ & $(0,14)$ \\
\hline \multirow[t]{2}{*}{ Constante } & $-2,12$ ** & 0,59 & $1,17 * *$ & $2,44 * * *$ \\
\hline & $(0,88)$ & $(0,44)$ & $(0,49)$ & $(0,50)$ \\
\hline
\end{tabular}

Se muestran coeficientes beta no estandarizados. Errores estándar entre paréntesis. ${ }^{* *} p<0,01 ;{ }^{* *} p<0,05,{ }^{*} p<0,1$.

los modelos) en el ZIMC en casi todo el espectro de peso, a excepción del p5 (bajo peso). Sexo femenino y residencia rural se asociaron a un menor ZIMC entre niños y niñas en el espectro de obesidad, pero no tuvieron un efecto significativo en ningún otro punto de la distribución de peso infantil.

Los resultados multivariados para los sujetos entre 4 y 6 años se resumieron en la tabla 3. En este caso, la interacción entre logaritmo del ingreso y educación materna del Modelo 2 fue significativa en el p50 (normopeso) y p95 (obesidad). Las figuras 1 y 2 ayudan a entender como actuaron estas variables en conjunto.

En ambos casos, hubo un cruce en los valores predichos del ZIMC, lo que indica que el efecto del ingreso fue negativo para niños cuyas madres tienen educación superior y positivo para hijos de madres sin educación superior. Así, en la Figura 1 (niños con normopeso), se observa que en el grupo de niños con madres de baja educación, al pasar del nivel más bajo al nivel más alto de ingreso familiar, el valor predicho de ZIMC cambia de 0,69 a 1,28 (un aumento de 0,59 desviaciones estándar); mientras que entre los niños con madres con educación superior, al pasar del nivel más bajo al nivel más alto de ingreso familiar, el valor predicho de ZIMC cambia de 1,38 a 0,95 (una disminución de 0,43 desviaciones estándar).

Respecto de los demás predictores, obesidad materna, igual que en el anterior tramo etario, tuvo un efecto positivo y grande sobre peso infantil, significativo en todo el espectro del ZIMC. La edad de los niños tuvo un efecto negativo y relativamente pequeño, no significativo entre los niños de peso normal (p50), pero sí en todo el resto del espectro del ZIMC. Sexo femenino también se asoció significativamente a un menor ZIMC, pero el efecto no fue robusto, dado que en los percentiles que era significativo en el Modelo 1 ( 55, p85 y p95) dejó de serlo en el Modelo 2, modelo en que el efecto de sexo femenino fue significativo en p50. Solo para niños con peso normal, la condición indígena o de ruralidad se asoció a mayor ZIMC. En el 


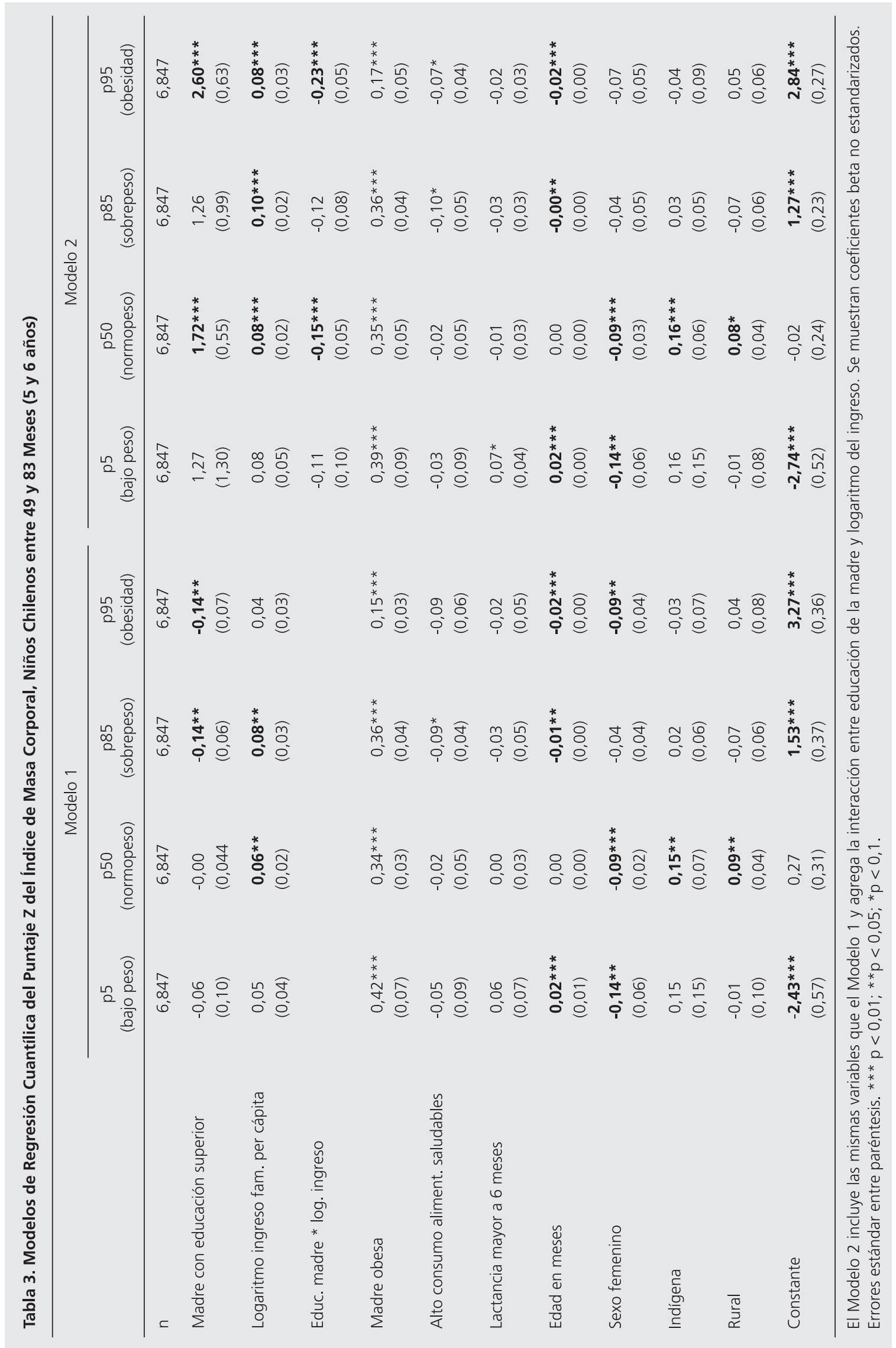


Modelo 2 y en el p5, la lactancia materna mayor a seis meses tuvo un efecto significativo, pero pequeño, sobre el peso infantil. Es decir, entre niños de bajo peso, lactancia prolongada se asoció a un ZIMC levemente superior.

\section{Discusión}

En este artículo se analizaron determinantes de ZIMC en niños obesos y no obesos, concentrando la atención en indicadores de NSE: educación materna e ingresos familiares. Consistente con la literatura mundial, el efecto con mayor magnitud en los modelos estimados fue tener una madre obesa ${ }^{24-28}$.

En relación con los determinantes socioeconómicos, investigación previa -en ciencias sociales y en ciencias de la salud- ha tendido a utilizar ingreso familiar y/o logro educativo como indicadores homologables e individualmente representativos de $\mathrm{NSE}^{13}$. Sin embargo, algunos estudios han comenzado a diferenciar estas variables. Por ejemplo, se ha constatado que el efecto de ingreso familiar en obesidad infantil puede ser tanto positivo como negativo. Por ejemplo, analizando IMC adolecente, Martin y cols. plantearon que mayor ingreso familiar aumentaba tanto la probabilidad de consumo de comidas procesadas y videojuegos como el acceso a comidas saludables y actividades físicas pagadas ${ }^{29}$. Además, el efecto de NSE sobre IMC depende del momento en la transición nutricional ${ }^{11,12}$. En países pobres, con altos niveles de desnutrición, la obesidad es más frecuente en familias de mayor NSE, pero la relación es inversa en países desarrollados ${ }^{8-12}$. En países de ingresos medios, existe evidencia de un efecto no lineal del nivel de desarrollo en el sobrepeso ${ }^{9}$.

Considerando estos hallazgos previos, este estudio analizó el efecto que tienen ingreso familar y logro educativo materno, por si solos, sobre el peso infantil y también su efecto combinado, mediante la interacción de ambos. Se examinó no solo en el peso infantil promedio, sino varios percentiles de peso, que cubren desde desnutricion hasta obesidad. Los resultados mostraron que tanto ingreso como educación tuvieron efectos significativos, pero en direcciones opuestas. Entre niños(as) entre 2 y 3 años, la asociación entre ZIMC y educación materna fue negativa, mientras que la asociación entre ZIMC e ingreso familiar fue positiva, y las dos variables no interactuaron. Entre niños(as) de 5 y 6 años, las dos variables interactuaron de manera significativa, tanto entre menores de peso normal como obesos. Así, entre hijos(as) de madres con educación superior, mayor ingreso se asoció a menor ZIMC, pero entre hijos(as) de madres con menos educación, mayor ingreso se asoció a mayor ZIMC. Esto nos lleva al debate respecto del efecto de la educación

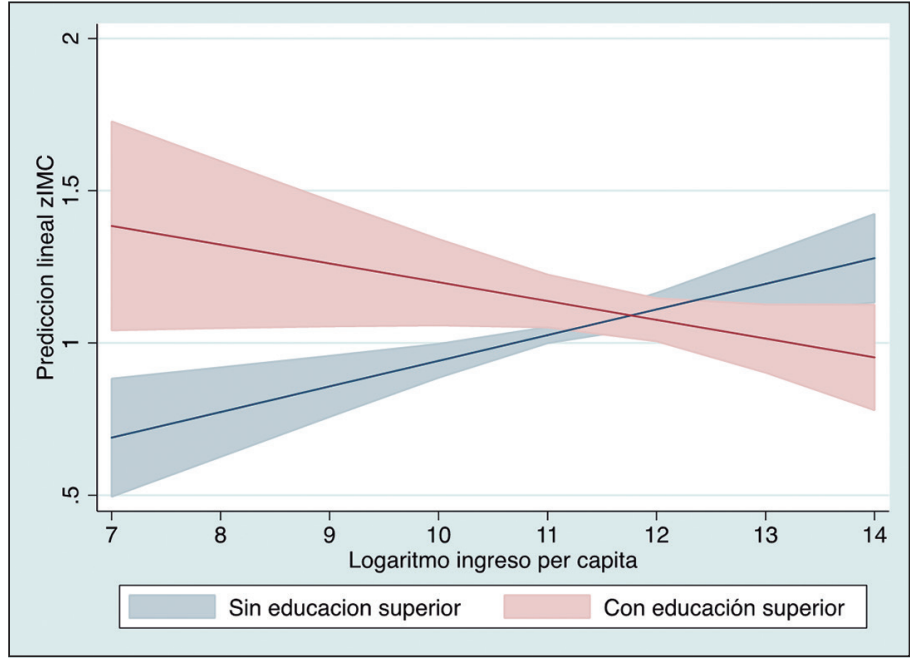

Figura 1. Valores predichos, ajustados, para el efecto de educación materna sobre el ZIMC, con intervalos de confianza al 95\%, p50. ZIMC: puntaje $Z$ para Índice de Masa Corporal. Los equivalentes en ingreso mensual per cápita del logaritmo del ingreso en el eje $X$ son: $7=\$ 1.097,8=\$ 2.981$, $9=\$ 8.103,10=\$ 22.026 .11=\$ 59.874,12=\$ 162.755,13=\$ 442.413 y$ $14=\$ 1.202 .604$.

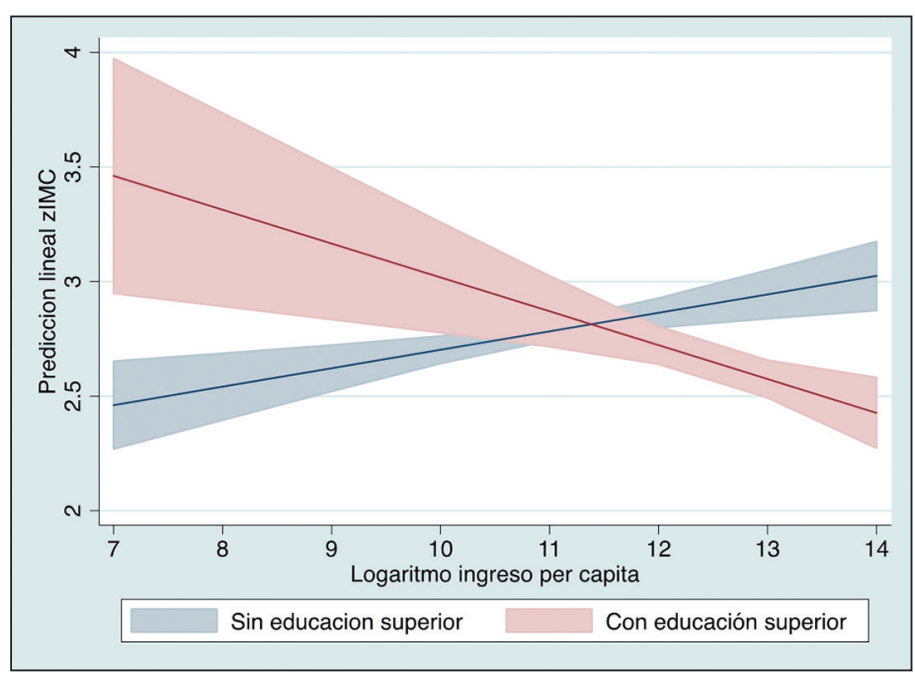

Figura 2. Valores predichos, ajustados, para el efecto de educación materna sobre el ZIMC, con intervalos de confianza al 95\%, p95. ZIMC: puntaje $Z$ para Índice de Masa Corporal. Los equivalentes en ingreso mensual per cápita del logaritmo del ingreso en el eje $X$ son: $7=\$ 1.097,8=\$ 2.981$, $9=\$ 8.103,10=\$ 22.026 .11=\$ 59.874,12=\$ 162.755,13=\$ 442.413 y$ $14=\$ 1.202 .604$.

en la salud: al constatar que las personas más educadas tienen en general mejores indicadores de salud, se discute si eso se debe a los mayores ingresos asociados a mayor educación o a un efecto propiamente educacional. Varios autores han subrayado que la educación mejora la capacidad de las personas para buscar y usar eficientemente información relacionada con la salud. Por ejemplo, Mirowsky y Ross (2003) han propuesto 
el concepto de "efectividad aprendida" para referirse a cómo al acumular más educación las personas ganan conocimientos, habilidades y recursos que les permiten optar por comportamientos que apuntan a un estilo de vida saludable ${ }^{30}$. Las preferencias alimentarias también podrían incidir. Por ejemplo, familias con movilidad social reciente -entre las cuales el ingreso ha crecido, pero el logro educacional puede mantenerse relativamente bajo- pueden consumir más comidas procesadas como marca de estatus ${ }^{31}$ o como parte de un ideario que privilegia lo industrial sobre lo casero. Por otro lado, si las madres tienen mayor educación, es más probable que se encuentren insertas en el mercado laboral, aportan un salario al ingreso familiar y por ello un mayor poder en la toma de decisiones sobre el uso de dichos recursos. Hay amplia evidencia que mujeres y hombres utilizan recursos familiares de diferentes maneras, con las mujeres destinando un mayor porcentaje al bienestar de los niños ${ }^{32}$. Esto se podría extrapolar a la compra de alimentos más saludables.

En el contexto chileno la transición demográficanutricional ocurrió en paralelo al acelerado crecimiento económico, y por ende se observa al mismo tiempo mayor poder adquisitivo, cambios en el ambiente alimentario (mayor disponibilidad de comidas procesada a precios accesibles) y en hábitos de preparación y consumo. En conclusión, estamos firmemente en lo que Popkin define como un patrón donde la así llamada dieta "occidental" causa enfermedades degenerativas ${ }^{10}$. Una adaptación que involucra un cambio en las conductas hacia dietas y hábitos saludables y prevenga los efectos adversos está aún pendiente.

Esta investigación hace varios aportes innovadores al estudio del peso infantil: el análisis de determinantes de peso en todo el espectro del IMC, desde la desnutrición hasta la obesidad, ejercicio inédito para el caso chileno; la consideración del efecto de educación materna y de ingresos familiares, tanto por separado como en conjunto, y la mirada al segmento etario de primera infancia.

A modo de conclusión, se establece la necesidad de continuar indagaciones sobre este tema, recomendando la desagregación de ingresos y educación en el análisis, como también investigación que indague en los mecanismos a través de los cuales estas dos variables afectan el peso infantil.

\section{Responsabilidades Éticas}

Protección de personas y animales: Los autores declaran que los procedimientos seguidos se conformaron a las normas éticas del comité de experimentación humana responsable y de acuerdo con la Asociación Médica Mundial y la Declaración de Helsinki.

Confidencialidad de los datos: Los autores declaran que han seguido los protocolos de su centro de trabajo sobre la publicación de datos de pacientes.

Derecho a la privacidad y consentimiento informado: Los autores han obtenido el consentimiento informado de los pacientes y/o sujetos referidos en el artículo. Este documento obra en poder del autor de correspondencia.

\section{Conflicto de intereses}

Las autoras declaran no tener conflicto de intereses.

\section{Agradecimientos}

Las autoras agradecen el apoyo de la Iniciativa Milenio, del Ministerio de Economía, Desarrollo y Turismo (Núcleo Milenio para el Estudio del Curso de Vida y la Vulnerabilidad) y de la Agencia Nacional de Investigación y Desarrollo ANID (Proyecto Fondecyt Iniciación No 1180717). Agradecen tambien el diligente trabajo de Valentina Jorquera y Valentina Moyano como asistentes de investigación.

\section{Referencias}

1. Albala C, Vio F, Kain J, Uauy, R. Nutrition transition in Chile: Determinants and consequences. Public Health Nutr. 2002;5(1a):123-8.

2. Informe Mapa Nutricional 2018. In. Santiago, Chile: Junta Nacional de Auxilio Escolar y Becas. JUNAEB; 2019.

3. Mönckeberg F, Muzzo S. La desconcertante epidemia de obesidad. Rev Chil Nutr. 2015; 42(1):96-102.

4. Rioseco R, Egaña Rojas D, Galvez
Esponiza P, Masferrer Riquelme D. Marco conceptual sobre los factores condicionantes de los ambientes alimentarios en Chile. In. Edited by Salud Md, Chile FdMUd. Chile; 2016.

5. World Health Organization. Nutrition Landscape Information System (Internet). Última visita: 30-05-2019. Disponible en: http://apps.who.int/nutrition/landscape/ report.aspx?iso $=\mathrm{chl}$

6. Celis-Morales C, Leiva A, Martínez M, Durán E, Labraña A, Petermann F, et al. Aumento del índice de masa corporal durante las últimas cuatro décadas en la población chilena: de la desnutrición a la obesidad. Rev Med Chile. 2017;145(10):1363-4.

7. Uauy R, Albala C, Kain J. Obesity Trends in Latin America: Transiting from Underto Overweight. J Nutr. 2001;131(3):893S-9S.

8. Caballero B. The Global Epidemic of Obesity: An Overview. Epidemiologic Rev 2007;29.

9. Caballero B: A nutrition paradoxunderweight and obesity in 
developing countries. N Engl J Med 2005;352:1514-6.

10. Popkin B, Adair LS, Ng SW: Then and Now: Global nutrition transition and the pandemic of obesity in developing countries. Nutrition Reviews 2011;70(1):3-21.

11. Popkin B: The Nutrition Transition in the Developing World. Development Policy Review 2003;21(5-6):581-97.

12. Popkin BM. Nutritional Patterns and Transitions. Popul Dev Rev. 1993;19(1):138-57.

13. Kain J, Herrera J, Lira M. Vulnerabilidad socioeconómica y obesidad en escolares chilenos de primero básico: comparación entre los años 2009 y 2013. Rev Chil Pediatr. 2018;86(06).

14. González-Zapata L, Carreño-Aguirre C, Estrada A, Monsalve-Alvarez J, Alvarez Stella L. Exceso de peso corporal en estudiantes universitarios según variables sociodemográficas y estilos de vida. Rev Chil Nutr. 2017;44(3):251-61.

15. Encuesta Longitudinal de Primer Infancia. Manual del Usuario Base de Datos Segunda Ronda Encuesta Longitudinal de Primera Infancia. Santiago: Universidad de Chile, Departamento de economía, Centro de micro datos; 2012.

16. Gelman A. Struggles with survey weighting and regression modeling. Statistical Science 2007;22(2):153-64.

17. Winship C, Radbill L. Sampling weights and regression analysis. Sociological Methods \& Research 1994;23(2):230-57.

18. De Onís M, Onyango A, Borghi E, Siyam A, Nashida C, Siekmann J. Elaboración de un patrón OMS de crecimiento de escolares y adolescentes. Bull World Health Organ. 2007;85(9):660-7.

19. Centers for Disease Control and Prevention (CDC). Growth Chart Training: Using the WHO Growth Charts (Internet). Última visita: 30-05-2019. Disponible en: https://www.cdc.gov/ nccdphp/dnpao/growthcharts/who/using/ index.htm

20. Marteleto L, Gama L, Dondero M, Letisha $\mathrm{E}$. The Weight of Inequality: Socioeconomic Status and Adolescent Body Mass in Brazil. Social Forces 2018;95(4).

21. Hao L, Naiman D. Quantile Regression. California: Sage Publications; 2007. 136p.

22. Kuczmarski R, Ogden C, GrummerStrawn L, Flegal K, Guo S, Wei R, et al. CDC growth charts: United States. Adv Data. 2000;314:1-27.

23. Rolland-Cachera M: Childhood obesity: current definitions and recommendations for their use. Int J Pediatr Obes. 2011;6:325-31.

24. Whitaker RC, Wright JA, Pepe MS, Seidel KD, Dietz WH. Predicting obesity inyoung adulthood from childhood and parental obesity. N Engl J Med. 1997;337(13):869-72.
25. Parsons TJ, Power C, Logan S, Summerbelt C. Childhood predictors of adult obesity: a systematic review. Int J Obes Relat Metab Disord. 1999;23(S8):S1-107.

26. Davison KK, Birch LL. Child and parent characteristics as predictors of change in girls' body mass index. Int J Obes. 2001;25(12):18-34.

27. Oken E. Maternal and child obesity: the causal link. Obstet Gynecol Clin North Am. 2009; 36(2):361-77.

28. Guillaume M, Lapidus L, Beckers F, Lambert A, Björntorp P. Familial trends of obesity through three generations: the Belgian-Luxembourg child study. Int J Obes Relat Metab Disord. 1995; 19:S5-9.

29. Martin MA, Frisco ML, Nau C, Burnett K. Social stratification and adolescent overweight in the United States: how income and educational resources matter across families and schools. Soc Sci Med. 2012;74(4):597-606.

30. Mirowsky J, Ross CE. Education, Social Status and Health. New York: Aldine De Gruyter; 2003.

31. Jacobs A, Richtel M. How Big Business Got Brazil Hooked on Junk Food. New York Times. 2017 Sept 16; Sect. Health.

32. Kenschaft L, Clark R. Gender Inequality in our Changing World: A comparative approach. New Yori: Routledge. 2016. 